\title{
Analysis of EEG rhythms under local sinusoidal ELF magnetic field exposure: An approach to neurofeedback enhancement on attention performance
}

\author{
Yasaman Zandi Mehran ${ }^{1 *}$, Mohammad Firoozabadi ${ }^{2}$, Reza Rostami $^{3}$ \\ ${ }^{1}$ Department of Biomedical Engineering, Science and Research Branch, Islamic Azad University, Tehran, Iran \\ ${ }^{2}$ Medical Physics Department, Tarbiat Modares University, Tehran, Iran \\ ${ }^{3}$ Department of Psychology and Educational Sciences, University of Tehran, Tehran, Iran \\ Email: ${ }^{*}$ zandi@srbiau.ac.ir
}

Received 4 July 2013; revised 12 August 2013; accepted 24 August 2013

Copyright (C) 2013 Yasaman Zandi Mehran et al. This is an open access article distributed under the Creative Commons Attribution License, which permits unrestricted use, distribution, and reproduction in any medium, provided the original work is properly cited.

\begin{abstract}
Although there is no consensus with respect to that if exposed Extremely Low Frequency Magnetic Field (ELF-MF) affects human brain activity for guidelines of brain management, there are some evidences related with human attention changes. Therefore, this study evaluates the effects of $45 \mathrm{~Hz}$ sinusoidal ELF (360 $\mu \mathrm{T})$ at $\mathrm{Cz}$ regions, cantered at dominant frequency using Electroencephalogram (EEG) analysis. The purpose was to extracte transient or permanent events as an index for new neurofeedback (NF) system improvement. Twenty-four healthy volunteers aged between 20 and 28 years of age were randomly assigned to one of two groups, which differed in the type of NF training concerning the exposed and non-exposed magnetic field effect on performance in attention tests during NF. Results indicate that theta and beta EEG rhythms variations in exposed group changed more significantly in comparison of traditional NF $(P<\mathbf{0 . 0 5})$. These results suggest a new design of NF system.
\end{abstract}

Keywords: Neurofeedback; Local Sinusoidal ELF; Attention

\section{INTRODUCTION}

An EEG is a recording of the electrical activity of the outer layer of the cerebral cortex. It is widely used to diagnose brain activity in clinical applications [1]. An EEG is an important safe clinical tool for diagnosing, monitoring neurological functions or disorders such as attention disorders [2-4]. Detecting and classifying transient or permanent changes by visual screening of the

\footnotetext{
${ }^{*}$ Corresponding author.
}

EEG record is a traditional operation and it doesn't require professionals. Over the past decade, the great related application has been made in the analysis of EEG signals (EEGs) for recognizing various functions of the brain to use as a feedback called Neurofeedback (NF) [5].

Neurofeedback is a brain training method which is related to electrical activity aspects of brain such as frequency, cerebral rhythm, and location or EEG activity extent. Neurofeedback is comprised of two aspects of active and passive. In active NF, there is a little external control factor (such as subject's intelligence role) which can affect the training factors, however, in the case of passive NF, volition is an inconspicuous role rather than the active one [6,7]. An active method is encountered with some problems such as numerous of training sessions. The other problem is the absence of a clinical tool as an effective index of the patients' status in training procedure.

Although, some developments have occurred in recent years, there are some instruments and technologies such as TMS, tDCS and etc., which are used in psychological treatment applications $[8,9]$. Therefore, if NF deficiencies removed using on-line and less side-effect and selective tool, NF could be more applicable for different purposes. Few papers confirm NF effectiveness in concentration, consciousness, attention even perception. However, the large number of sessions with medium effectiveness demonstrates that the traditional NF method does not give the required efficiency and capability yet [10-15].

In this study, it is aimed to modify a traditional NF procedure. For example, decrement of theta to beta ratio obtained from Fourier analysis is relevant to attention increment in human $[16,17]$, therefore, observation of the changes in this ratio during NF can warn the operator or physician about the training procedure; however, the effect of subjects training characteristics and the numerous 
training sessions still remains. Therefore, this study goal leads to a proper method with more efficiency and desired changes in brain.

It is proven that Extremely Low Frequency Magnetic Field $(0-300 \mathrm{~Hz}$, intensity $<500 \mu \mathrm{T})$ called ELF-MF affects human brain activity based on resultant evidence in some studies. Usually, Helm Holts coils are used to magnetic field expose. Briefly, Cook et al. [18] reviewed reported effects on different intensity and frequency of the magnetic field. In fact, in that research it was aimed to examine the surrounding MF on body, especially on the brain and its diseases such as epilepsy.

Some of the researches believe that at some frequencies, ELF MF causes amplification of the frequency as the exposure field's one, in EEG signals which is called resonance phenomena $[19,20]$. There are some researches on effects of very weak alternating magnetic fields upon living organisms and related variations in the ion concentrations within the cells when the frequency of the applied field matches the angular frequency and resonance phenomena investigations. Some papers focused on the cyclotron or Larmor frequency mechanism by which biological systems become sensitive to small static and resonating magnetic fields and existence of a resonating effect on ions. Table 1 summarized some of the ELF-MF cellular interactions and mechanism.

On the other side, exploring magnetic field effects on perception, concentration, cognition (consciousness level) and electrophysiology of the neurons especially within the brain are unknown yet, however, as described in this study, the MF effect is considerably effective on brain which can be used to the improvement of NF system. Some few researchers have focused on MF effects on electrical activity of brain, where the cognitive and per- ceptual effects of MF exposure were explored [21-25].

It seems that by emphasizing on the ELF MF effect on EEG rhythms, a novel passive NF based on implementation of magnetic field is designed and explored. By local sinusoidal exposure, more selective and less side effects may be achieved. In this study, processing of affected EEG signals in the presence of LSELF-MF is utilized in implementation of new idea as an off-line or even on-line method to achieve a new more applicable passive NF system.

\section{MATERIALS AND METHODS}

\subsection{Local Sinusoidal ELF-MF Exposure}

This key idea can develop traditional NF to modified NF we called Neuro-LSELF-MF which its results in pursuing oriented method to affect the brain activity to improve NF system. Shafiei et al. [26-29] found significant decrease in theta $(5-8 \mathrm{~Hz})$ rhythm after $45 \mathrm{~Hz}$ SLELF-MF exposure in central region of brain.

One of the purposes in NF training is an attempt at examining whether NF training can positively influence cognitive performance such as attention. Some researches show $[5,16,30]$ that enhance beta $(15-18 \mathrm{~Hz})$ whilst simultaneously inhibiting theta $(4-7 \mathrm{~Hz})$ and high beta $(22-30 \mathrm{~Hz})$ result in attention level after 10 sessions.

So the key idea of this study is SLELF-MF discussed effects ( $45 \mathrm{~Hz}$ at $\mathrm{Cz}$ region) on EEG rhythms in a direction that increment in attention level by affecting theta and beta rhythms, occurred. For more analysis performance and subjects scoring, we used Fast Fourier Transform (fft) and other more precise investigations which are not discussed in this paper. Finally, we demonstrate a new NF improved system.

Table 1. Summary of ELF-MF exposure interaction upon ions and cells and neurons.

\begin{tabular}{|c|c|}
\hline Study & Mechanism \\
\hline $\begin{array}{l}\text { Capone et al. } \\
\text { (Capone, F., 2009) }\end{array}$ & Enhancement in cortical excitatory neurotransmission \\
\hline $\begin{array}{l}\text { De Ninno et al. } \\
\text { (De Ninno, A., 2008) }\end{array}$ & $\begin{array}{l}\text { Superoxide radical's generation by a weak field having the Larmor frequency }\left(\mathrm{f}_{\mathrm{L}}\right) \text { of } \mathrm{Fe}^{2+} \text { while the SOD1 kinetics is sensibly } \\
\text { reduced by exposure to a weak field having the frequency } \mathrm{f}_{\mathrm{L}} \text { of } \mathrm{Cu}^{2+} \text { ion }\end{array}$ \\
\hline $\begin{array}{l}\text { Gerardi et al. } \\
\text { (Gerardi, G., 2008) }\end{array}$ & Affecting parameters like blood glucose and fatty acid metabolism \\
\hline $\begin{array}{l}\text { Manikonda et al. } \\
\text { (Manikonda, P.K., 2007) }\end{array}$ & $50 \mathrm{~Hz}$ ELF-MF Modify in NMDA receptor function mediated by alteration of $\mathrm{Ca}^{2+}$ signalling in rat hippocampus \\
\hline $\begin{array}{l}\text { McFarlane et al. } \\
\text { (McFarlane, E.H., 2000) }\end{array}$ & Changes in neuritis outgrowth but not in cell division induced by ELF \\
\hline $\begin{array}{l}\text { Piacentini et al. } \\
\text { (Piacentini, R., 2008) }\end{array}$ & $\begin{array}{l}\text { Increases the expression and function voltage-gated } \mathrm{Ca}^{2+} \text { channels that } \mathrm{Ca}^{2+} \text { influx through } \mathrm{Ca}(\mathrm{v}) 1 \text { channels that plays a key } \\
\text { role in promoting the neuronal differentiation of neural stem/progenitor cells (NSCs) }\end{array}$ \\
\hline $\begin{array}{l}\text { Pirozzoli et al. } \\
\text { (Pirozzoli, M.C., 2003) }\end{array}$ & Modify gene-expression in neuron-like cells \\
\hline $\begin{array}{l}\text { Salamino et al. } \\
\text { (Salamino, F., 2006) }\end{array}$ & Weak magnetic fields strongly decreases the calpain catalytic activity \\
\hline
\end{tabular}




\subsection{Subjects}

Twenty-four volunteers ( 6 female and 18 male) aged between 20 and 28 years (mean age of $23.29 \pm 1.94$ years) in good physical and mental health (no women attend during Women's menstruation period to avoid interference of the hormonal fluctuations of the menstrual cycle) were attend in the study. The participants were recruited through advertisements at the Bioelectrical Lab of Biomedical Engineering Department. The ethics committee of the University approved the protocol and all volunteers provided informed consent of ELF. Subjects participated in a 30 minutes session of testing between 8.30 and 9 A.M. or 9.30 and 10 A.M. or 10.30 and 11 A.M. or 11.30 and 12 A.M. Each subject underwent to $10 \mathrm{~min}-$ utes NF attendance session with at least two day intervals both in exposure and sham groups.

\subsection{Experimental Design}

The magnetic exposure system consisted of a circular coil. The electrode wire passed through the opening Plexiglas ring and the electrode was centered inside the coil. The signal generator was made to produce the output signal. The coil characteristics measured by EDC-1630 GIGITAL L.C.R METER, EQ model $(\mathrm{L}=12.73 \pm 0.025$ $\mathrm{mH}, \mathrm{C}=0.9846 \pm 0.001 \mu \mathrm{F}, \mathrm{R}=16.13 \pm 0.04 \Omega$ ). Considering the coil properties and low frequency range of signal generator $(0.5$ to $100 \mathrm{~Hz})$, inductance effects is ambiguous. After turning off the signal generator, the current of the coil rapidly damps.

Tesla meter (TRIAXIAL ELF MAGNETIC FIELD METER, TES-1394, U.S. Pat. No. Des. 446, 135) at 1.2 $\mathrm{cm}$ below the Plexiglas ring at the axis showed the intensity of ELF-MF as $360 \mu \mathrm{T}$ as rms. For the existence skull and its beneath layers, there is a $1.2 \mathrm{~cm}$ distance between stimulated point and target effect point. Thus adjustment of signal generator settings was so that the exposure intensity set at $360 \mu \mathrm{T}$ at the desired stimulated point. For this purpose, we set the coil on the piece of dead skull and then measured the desired intensity as rms LSELFMF.

The EEG recorder device (FlexComp Infinity, Thought Technology Ltd, Model: SA7550) has 2 channels for EEG recording and an elastic band, glued to the scalp on both sides, holds gold active electrodes (EEG-Z T.T.Ltd, Model: SA9305, Z5417) and coil both, which are placed on $\mathrm{Cz}$. The resistance between electrode and skin of head is below $1 \mathrm{k} \Omega$ (measurement abilities of FlexComp Infinity NF system). The 10/20 IS of electrode placement is used and the actual placement has a common reference electrode placed at the left ear lobe and is grounded to the right ear lobe using color coded ear clips. For this study purposes, sinusoidal local ELF exposed as 2 seconds ON and 3 seconds OFF (Duty Cycle $=40 \%$ ).
In this study, the noisy EEG signal is due to the LSELF exposure from the points of current propagation of the ELF coils, and consequently the collected data has a very low signal to noise ratio at the exposure times. This phenomena affect the analysis and the training too. To eliminating, the LSELF exposure signal is recorded with the second channel of EEG electrode, placed on an aluminum shielded wire above the coil. The two channels data can synchronously be analyzed directly in LabVIEW (National Instruments; Austin, TX, 2010). A dedicated algorithm implemented in LabVIEW coded by Matlab (R2012a, Ver. 7.17.0.739) for on-line de-noising and analyzing of the recorded EEG data both in exposed and not exposed times, for real-time processing. By threshold detection method, when LSELF exposure starts, subjects didn't receive any feedback. Although this phenomena appears only in on times of NF training but in off times, it is possible to record EEGs.

\subsection{Procedure}

The EEG monitoring was done by a mono-polar electrode placement at $\mathrm{Cz}$, with two ear electrodes providing a reference and ground. Each training session lasted for 6 minutes (without considering LSELF-MF exposed times in both group) with the objective reinforcement to inhibit theta and high beta activities while increasing beta waves. Each of 16 subjects of exposure group, exposed for 2-sec epochs exhibited altered brain electrical activity during the time of stimulation, but in sham group only the coil was on the head with no exposure. The sinusoidal local ELF exposed as 2 seconds ON and 3 seconds OFF.

The EEG is then sent to LabVIEW via a USB port to a laptop under window 7. Data acquisition sampling rate was $256 \mathrm{~Hz}$ and a band pass filtration was performed from $2 \mathrm{~Hz}$ to $50 \mathrm{~Hz}$ with a $50 \mathrm{~Hz}$ Notch filter. For each 1 sec cycle, a LabVIEW program detected whether the ELF signal exceeded a threshold. The system is designed such that an ELF signal corresponds to the exposure times appears and this signal is condition in this way so that can keep the device real-time and eliminate the signal summated with ELF exposure. The ELF signal which is synchronizing recorded by second channel, is then used to eliminate the related EEGs under exposure, and thus damped by any feedback sending to subject. But due to windowing of a signal that causes its spectrum and frequency specification changes and to develop a non-zero values at frequencies other than its real spectrum, the 256 samples hanning window used to analyzing the EEGs applied by LabVIEW.

\section{RESULTS AND DISCUSSIONS}

EEG rhythms were saved analyzed with a repeatedmeasure analysis of variance (ANOVA) with three within- 
factor variables (exposure condition, session number and trial state). Two states were considered for exposure condition: SHAM (only NF training) and LSELF-MF EXPOSURE (NF training and LSELF-MF exposure simultaneously). As to the trial state, three levels PRE, DURING and POST were considered for some EEG rhythms; for what concerns theta: $4-8 \mathrm{~Hz}$; beta: 15 - 18. The information is contained in certain features of the EEG such as the absolute power analyzed by fft method. For EEG rhythms parameters, ANOVA with one within-factor (i.e. exposure condition contains Sham and Exposure; two states: sham and magnetic) was applied. All analyses were run with the statistical IBM SPSS Statistics ver.21 software. A statistical significant effect was defined when the level of significance was $P<0.05$.

Using between-group comparisons there was no significant difference between the groups at pre-testing for age (Real mean for Exposed group $=22.8750$ (1.8930) and Real mean for Sham group $=24.1225$ (1.8851) years old).

To compare the difference of EEG in PRE and POST LSELF-MF, rhythms Fourier estimation is used. The Kolmogorov-Smirnov test showed that EEG rhythms belong to normal distribution, so t-test across the statuses of PRE, DURING and POST were adopted.

Figure 1 shows both the EEG rhythms of PRE, DURING and POST of both groups, and there is an obvious

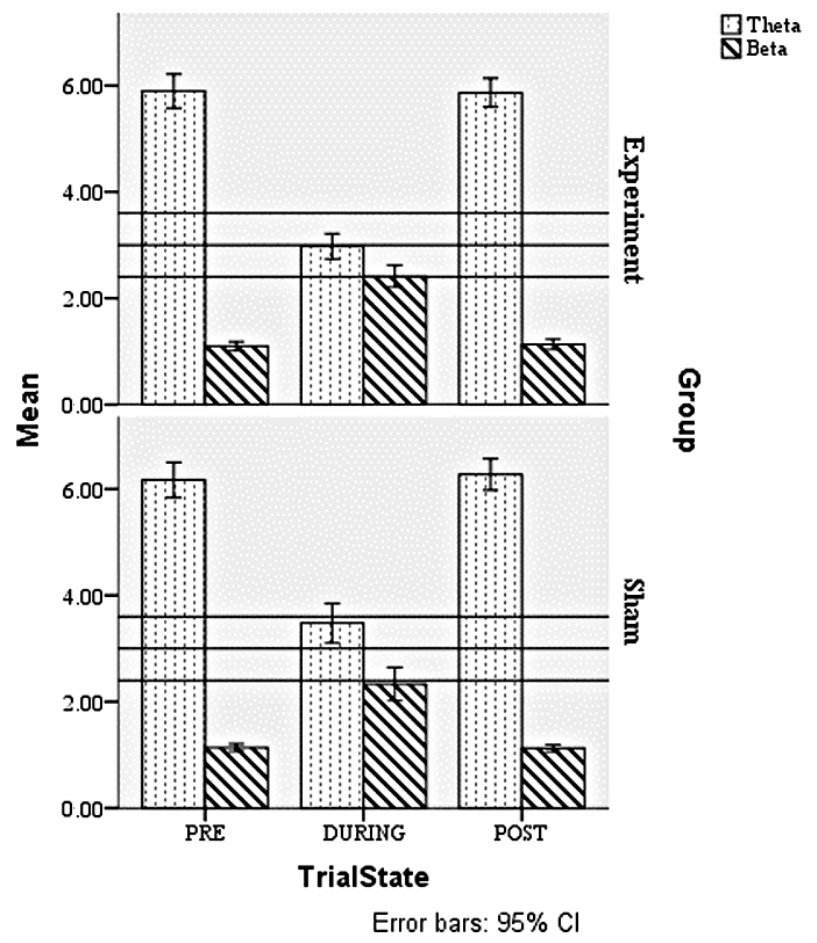

Figure 1. The mean amplitude for the training frequency of theta $(4-8 \mathrm{~Hz})$; beta $(15-18 \mathrm{~Hz})$; EEG rhythms for each of three trial state as PRE, DURING and POST states of two groups. difference between DURING in comparison of PRE and POST in two groups. As the results show, both traditional NF (sham) and LSELF-MF exposure (experiment) in during state, result in decrement in theta and increment beta, both.

It is apparent that theta and beta of EEG rhythms of exposed group are much different with sham state. As illustrated in this figure, there is more theta decrement in comparison of sham group when LSELF-MF is applied. The beta increment of experiment group is more than the sham group, but not as the same scale of theta decrement changes. It seems that the $45 \mathrm{~Hz}$ LSELF-MF has a selective effect on theta damping.

Significant differences appeared in DURING of theta band $(P<0.05)$, DURING of beta band $(P<0.05)$. Paired sample t-test showed that LESEL-MF during NF significantly changed EEG rhythms of beta and theta band. However, sham group impact these parameters of EEG significantly $(P<0.05)$ too. In addition, the effect of LSELF-MF differed significantly from sham training on some of EEG band such as theta and beta as described at Table 2 .

Results suggested that EEG rhythms correlated with attention activity upon LSELF-MF of scales sham group were much changed than those in sham group. The amplitude of EEG rhythms of most subjects is changed after new designed method. Thus the conclusion is that NF affects EEG but using LSELF-MF during NF has certain suppressive effects on brain.

\section{CONCLUSION}

There is evidence that ELF magnetic waves can affect brain waves but no linear correlation is found between the intensities, frequencies of electromagnetic waves yet and its effects on EEG records [18,22,26-28], therefore, designing an efficient and applicable system with a traditional NF form can be effective in undertaking these waves effects. By using regional exposure and stimulation of some regions of human head, the nonlinear effects of stimulation can be removed. Based on theoretical view point of magnetic induction resonance effects on cerebral waves $[19,20]$, it is proposed to systematically impose meaningful changes on EEG signal, either actively or inactively, which needs more studies. To eliminate the effectiveness of individuals on results, ELF can be used, which leads to decreasing of treatment sessions and achieving a desired status. Thus, simultaneously using ELF in a system with proper NF protocol may increase the efficiency of this method. This strategy of using composed methods and theoretical viewpoint in this project is to eliminate these deficiencies. In order to speed up and improve the method by the results of ELF MF exposure, the role of individual volition in treatment process is decreased; therefore, the treatment sessions 
Table 2. The EEG rhythms variations of both groups.

\begin{tabular}{|c|c|c|c|c|c|c|c|c|}
\hline & & & \multicolumn{6}{|c|}{ TrialState } \\
\hline & & & \multicolumn{2}{|r|}{ PRE } & \multicolumn{2}{|r|}{ DURING } & \multicolumn{2}{|r|}{ POST } \\
\hline & & & Mean & Standard Deviation & Mean & Standard Deviation & Mean & Standard Deviation \\
\hline \multirow{3}{*}{ Group } & \multirow{2}{*}{ Experiment } & Theta & 5.90 & 2.01 & 2.98 & 1.49 & 5.87 & 1.69 \\
\hline & & Beta & 1.15 & 0.58 & 2.42 & 1.30 & 1.18 & 0.61 \\
\hline & Sham & Theta & 6.17 & 1.49 & 3.49 & 1.62 & 6.27 & 1.32 \\
\hline
\end{tabular}

Note: Values in the same row and subtable not sharing the same subscript are significantly different at $\mathrm{p}<0.05$ in the two-sided test of equality for column means. Cells with no subscript are not included in the test. Tests assume equal variances. ${ }^{1}{ }^{1}$ Tests are adjusted for all pairwise comparisons within a row of each innermost subtable using the Bonferroni correction.

might be decreased as it is expected.

But the results show that LSEELF-MF did not lead to resonance phenomena in exposed region. The exposure of local ELF MF leads to blocking of some EEG rhythms versus exciting some others. Although no intensive and systematic effect was observed, MF's estimating frequency has considerable influence on cerebral signals which can be designed and evaluated in protocols to treat some psychological diseases. Therefore, the design of an applicable NF system is not substantiated. This difficulty is caused by wide stimulation of other works (Cook CM, 20-09). For the case of local exposure and limited stimulation of brain, this problem is removed. It is hoped that meaningful oriented changes on EEG signal are systematically implemented in future works.

\section{ACKNOWLEDGEMENTS}

Special thanks for Thought Technology Ltd. and Inside Sales and Marketing Managers, Didier Combatalade and Helen Mavros for helpful information and comments.

\section{REFERENCES}

[1] B. Allison, C. Brunner, V. Kaiser, G. Müller-Putz, C. Neuper and G. Pfurtscheller (2010) Toward a hybrid braincomputer interface based on imagined movement and visual attention. Journal of Neural Engineering, 7, Article ID: 026007.

[2] Lansbergen, M.M., van Dongen-Boomsma, M., Buitelaar, J.K. and Slaats-Willemse, D. (2011) ADHD and EEGneurofeedback: A double-blind randomized placebo-controlled feasibility study. Journal of Neural Transmission, 118, 275-284.

http://dx.doi.org/10.1007/s00702-010-0524-2

[3] Thompson, M. and Thompsonm L. (2005) Neurofeedback Intervention for Adults with ADHD. Journal of Adult Development, 12, 123-130. http://dx.doi.org/10.1007/s10804-005-7028-6

[4] Thompson, M. and Thompsonm L. (2003) The neurofeedback book: An introduction to basic concepts in applied psychophysiology.

[5] Ochs, L. (2006) The LOW ENERGY NEUROFEEDBACK SYSTEM (LENS): Theory, background, and in- troduction. Journal of Neurotherapy, 10, 5-39.

http://dx.doi.org/10.1300/J184v10n02 02

[6] Ochs, L. (2006) Comment on the treatment of fibromyalgia syndrome using low-intensity neurofeedback with the flexyx neurotherapy system: A randomized controlled clinical trial; or, how to go crazy over nearly nothing. Journal of Neurotherapy, 10, 59-61.

http://dx.doi.org/10.1300/J184v10n02 04

[7] Gladwin, T.E., den Uyl, T.E., Fregni, F.F. and Wiers, R.W. (2012) Enhancement of selective attention by tDCS: Interaction with interference in a Sternberg task. Neuroscience Letters, 512, 33-37. http://dx.doi.org/10.1016/j.neulet.2012.01.056

[8] Weaver, L., Rostain, A.L., Mace, W., Akhtar, U., Moss E. and O'Reardon, J.P. (2012) Transcranial magnetic stimulation (TMS) in the treatment of attention-deficit hyperactivity disorder in adolescents and young adults: A pilot study. Journal of ExtraCorporeal Technology, 28, 98103.

[9] Hanslmayr, S., Sauseng, P., Doppelmayr, M., Schabus M. and Klimesch, W. (2005) Increasing individual upper alpha power by neurofeedback improves cognitive performance in human subjects. Applied Psychophysiology and Biofeedback, 30, 1-10. http://dx.doi.org/10.1007/s10484-005-2169-8

[10] Herrmann, C.S. and Knight, R.T. (2001) Mechanism of human attention: Event-related potentials and oscillations. Neuroscience \& Biobehavioral Reviews, 25, 465-476. http://dx.doi.org/10.1016/S0149-7634(01)00027-6

[11] Klimesch, W. (1999) EEG alpha and theta oscilliations reflect cognitive and memory performance: A review and analysis. Brain Research Reviews, 29, 169-195. http://dx.doi.org/10.1016/S0165-0173(98)00056-3

[12] Lubar, J.F. (1997) Neocortical dynamics: Implications for understanding the role of neurofeedback and related techniques for the enhancement of attention. Applied Psychophysiology and Biofeedback, 22, 111-126. http://dx.doi.org/10.1023/A:1026276228832

[13] Thompson, M. and Thompsonm L. (2006) Improving attention in adults and children: Differing electroencephalograhy profiles and implications for training. Biofeedback, 34, 99-105.

[14] Zoefel, B., Huster, R.J. and Herrmann, C.S. (2011) Neurofeedback training of the upper alpha frequency band in EEG improves cognitive performance. Neuroimage, 54, 1427-1431. 
http://dx.doi.org/10.1016/j.neuroimage.2010.08.078

[15] Vernon, D., Egner, T., Cooper, N., Compton, T., Neilands, C., Sheri, A. and Gruzelier, J. (2003) The effect of training distinct neurofeedback protocols on aspects of cognitive performance. International Journal of Psychophysiology, 47, 75-85.

http://dx.doi.org/10.1016/S0167-8760(02)00091-0

[16] Vernon, J.D. (2005) Can neurofeedback training enhance performance? An evaluation of the evidence with implications for future research. Applied Psychophysiology and Biofeedback, 30, 347-364.

http://dx.doi.org/10.1007/s10484-005-8421-4

[17] Cook, C.M., Thomas, A.W. and Prato, F.S. (2002) Human electrophysiological and cognitive effects of exposure to ELF magnetic and ELF modulated RF and microwave fields: A review of recent studies. Bioelectromagnetics, 23, 144-157.

http://dx.doi.org/10.1002/bem.107

[18] Bell, G.B., Marino, A.A. and Chesson, A.L. (1994) Frequency-specific responses in the human brain caused by electromagnetic fields. Journal of the Neurological Sciences, 123, 26-32.

http://dx.doi.org/10.1016/0022-510X(94)90199-6

[19] Bell, G.B., Marino, A.A. and Chesson, A.L. (1994) Frequency-specific blocking in the human brain caused by electromagnetic fields. Neuroreport, 5, 510-512. http://dx.doi.org/10.1097/00001756-199401120-00036

[20] Bell, G.B., Marino, A.A. and Chesson, A.L. (1991) Alterations in brain electrical activity caused by magnetic fields: Detecting the detection process. Electroencephalography and Clinical Neurophysiology, 3, 389-397.

[21] Cook, C.M., Saucier, D.M., Thomas, A.W. and Prato, F.S. (2009) Changes in human EEG alpha activity following exposure to two different pulsed magnetic field sequences. Bioelectromagnetics, 30, 9-20. http://dx.doi.org/10.1002/bem.20434

[22] Liu, T., Wang, S., He, L. and Ye, K. (2008) Chronic exposure to low-intensity magnetic field improves acquisition and maintenance of memory. NeuroReport, 19, 549552. http://dx.doi.org/10.1097/WNR.0b013e3282f8b1a0

[23] Podd, J., Abbott, J., Kazantzis, N. and Rowland, A. (2002) Brief exposure to a $50 \mathrm{~Hz}, 100$ micro T magnetic field: Effects on reaction time, accuracy, and recognition memory. Bioelectromagnetics, 23, 189-195. http://dx.doi.org/10.1002/bem.10004

[24] Vázquez-García, M., Elías-Viñas, D., Reyes-Guerrero, G., Domínguez-González, A., Verdugo-Díaz, L. and GuevaraGuzmán, R. (2004) Exposure to extremely low-frequency electromagnetic fields improves social recognition in male rats. Physiology \& Behavior, 82, 685-690. http://dx.doi.org/10.1016/j.physbeh.2004.06.004

[25] Shafiei, S.A., Firoozabadi, S.M., Rasoulzadeh Tabatabaie, K. and Ghabaee, M. (2010) EEG changes during exposure to extremely low frequency magnetic field on a small area of brain. Koomesh, 12, 167-174.

[26] Shafiei, S.A., Firoozabadi, S.M., Rasoulzadeh Tabatabaie, K. and Ghabaee, M. (2011) Investigation of resonance effect caused by exposure of local extremely low frequency magnetic field on brain signals. Qom University
Medical Science Journal, 5, 53-60.

[27] Shafiei, S.A., Firoozabadi, S.M., Rasoulzadeh Tabatabaie, K. and Ghabaee, M. (2012) Study of the frequency parameters of EEG influenced by zone-dependent local ELF-MF exposure on the human head. Electromagnetic Biology and Medicine, 31, 11-21.

http://dx.doi.org/10.3109/15368378.2011.624658

[28] Shafiei, S.A., Firoozabadi, S.M., Rasoulzadeh Tabatabaie, K. and Ghabaee, M. (2012) Investigation of EEG subbands under ELF MF for psychological disease (in Persian language). Iranian Psychological Association Congress, 9, 141-152.

[29] Egner, T. and Gruzelier, J.H. (2004) EEG biofeedback of low beta band components: Frequency-specific effects on variables of attention and event-related brain potentials. Clinical Neurophysiology, 115, 131-139. http://dx.doi.org/10.1016/S1388-2457(03)00353-5

[30] Capone, F., Dileone, M., Profice, P., Pilato, F., Musumeci, G., Minicuci, G., Ranieri, F., Cadossi, R., Setti, S., Tonali, P.A. and Di Lazzaro, V. (2009) Does exposure to extremely low frequency magnetic fields produce functional changes in human brain? Journal of Neural Transmission, 116, 257-265. http://dx.doi.org/10.1007/s00702-009-0184-2

[31] De Ninno, A., Prosdocimi, M., Ferrari, V., Gerardi, G., Barbaro, F., Badon, T. and Bernardini, D. (2008) Effect of ELF e.m. fields on metallo protein redox-active sites.

[32] G. Gerardi, A. De Ninno, M. Prosdocimi, V. Ferrari, F. Barbaro, S. Mazzariol, D. Bernardinil and G. Talpo. (2008) Effects of electromagnetic fields of low frequency and low intensity on rat metabolism. BioMagnetic Research and Technology, 6, 3. http://dx.doi.org/10.1186/1477-044X-6-3

[33] Manikonda, P.K., Rajendra, P., Devendranath, D., Gunasekaran, B., Channakeshava Aradhya, R.S., Sashidhar, R.B. and Subramanyam, C. (2007) Influence of extremely low frequency magnetic fields on $\mathrm{Ca}^{2+}$ signaling and NMDA receptor functions in rat hippocampus. Neuroscience Letter, 413, 145-149. http://dx.doi.org/10.1016/j.neulet.2006.11.048

[34] McFarlane, E.H., Dawe, G.S., Marks, M. and Campbell, I.C. (2000) Changes in neurite outgrowth but not in cell division induced by low EMF exposure: influence of field strength and culture conditions on responses in rat $\mathrm{PC} 12$ pheochromocytoma cells. Bioelectrochemistry, 52, 23-28. http://dx.doi.org/10.1016/S0302-4598(00)00078-7

[35] Piacentini, R., Ripoli, C., Mezzogori, D., Azzena G.B. and Grassi, C. (2008) Extremely low-frequency electromagnetic fields promote in vitro neurogenesis via upregulation of $\mathrm{Ca}(\mathrm{v}) 1$-channel activity. Journal of Cellular Physiology, 215, 129-139. http://dx.doi.org/10.1002/jcp.21293

[36] Pirozzoli, M.C., Marino, C., Lovisolo, G.A., Laconi, C., Mosiello, L. and Negroni, A. (2003) Effects of $50 \mathrm{~Hz}$ electromagnetic field exposure on apoptosis and differentiation in a neuroblastoma cell line. Bioelectromagnetics, 24, 510-516. http://dx.doi.org/10.1002/bem.10130

[37] Salamino, F., Minafra, R., Grano, V., Diano, N., Mita, D.G., Pontremoli, S. and Melloni, E. (2006) Effect of extremely low frequency magnetic fields on calpain activa- 
tion. Bioelectromagnetics, 50, 27-43.

[38] Doehnert, M., Brandeis, D., Straub, M., Steinhausen, H.C. and Drechsler, R. (2008) Slow cortical potential neuronfeedback in attention deficit hyperactivity disorder: Is there neurophysiological evidence for specific effects? Journal of Neural Transmission, 115, 1445-1456. http://dx.doi.org/10.1007/s00702-008-0104-x 